\title{
An efficient approach for assessing the seismic soil structure interaction effect for the asymmetrical pile group
}

\author{
Pallavi Badry ${ }^{1} \cdot$ Neelima Satyam ${ }^{1}$
}

Received: 20 June 2015/Accepted: 15 February 2016/Published online: 10 May 2016

(C) Springer International Publishing Switzerland 2016. This article is published with open access at Springerlink.com

\begin{abstract}
All of the civil engineering structures involve some type of structural element which is in direct contact with soil. To estimate the accurate response of the superstructure it is necessary to consider the response of the soil supporting structure, and is well explained in the soil structure interaction analysis. Many attempts have been made to model the SSI problem numerically; however the soil nonlinearity, foundation interfaces and boundary conditions make the problem more complex and computationally costlier. To overcome this problem the attempt has been made to optimize the computational efficiency by applying the equivalent pier method for the deep foundation system. In this research paper the L-shape 11 storey building supported by a pile foundation with homogeneous local soil condition is analyzed for dynamic loading including the SSI effect. The significance of the SSI effect has been studied by comparing the responses of the system for fixed base and flexible base condition. A new approach has been proposed to provide simplicity in SSI modeling and reduce the computational cost (both memory and time wise). The approach includes the applicability of the equivalent pier method for the asymmetrical pile groups system, including SSI effect of the pile foundation system. The approach is validated for group effect and found that equivalent pile method can successfully be adopted and helps to reduce the computational cost of SSI problem. To understand the applicability of EPM approach, the
\end{abstract}

Neelima Satyam

neelima.satyam@iiit.ac.in

Pallavi Badry

pallavi.ravishankar@ research.iiit.ac.in

1 Geotechnical Engineering Laboratory, International Institute of Information Technology, Hyderabad, India parametric study has been carried out for different input of earthquakes and soil types. In accordance with this the three distinct earthquakes, including 1995 Chamba $(\mathrm{M}=4.9), 1999$ Uttarkashi $(\mathrm{M}=6.9)$ and 2001 Bhuj $(\mathrm{M}=7.7)$ and soil types including cohesive, cohesionless and C-Phi soils have been considered for SSI analysis. The study observed that, earthquake magnitude and soil type shows the major impact on the response of the SSI system.

Keywords DSSI · Asymmetrical building · Soil pile interaction - Asymmetrical pile groups $\cdot$ Equivalent pier method

\section{Introduction}

Every important structure, including nuclear power plant and multistory buildings, founded on the soft strata need to analyze by considering the interaction effect. In order to design such complex system closer to the reality, the nonlinear response is needed to estimate. Substantial research attempts have been carried out to investigate the kinematic seismic behavior of single piles and pile groups including linear and nonlinear soil behavior. In all the above reviews it has been revealed that the tall, shear type and pile supported symmetrical buildings supported on homogeneous, heterogeneous, linear, elastic, nonlinear medium have been attempted very well. But the dynamic structure, soil structure interaction of pile supported asymmetrical frames is not being addressed so far. As asymmetrical building is one of very common types of buildings, needed to focus to analyze accurately [12].

Most design codes have provided detailed provisions for structure asymmetry and torsion-resistant design [5]. However, the destruction of numerous asymmetric 
buildings in earthquakes like Bucharest 1977, Mexico City 1985; Kobe 1995 and Bhuj 2001 made researchers realize that soil structure interaction (SSI) can substantially change the seismic performance of asymmetric structures. Several damages have been evidenced during earthquakes due to incomprehension of soil structure interaction effect in design of structure and foundation system [30]. In the 1985 Mexico earthquake high rise building collapsed due to the partial bearing capacity failure of foundation soil. It has been reported that this earthquake was particularly destructive to the unbraced buildings founded on soft soils due to the increase in a fundamental time period of soil from about $1.0 \mathrm{~s}$ to nearly $2.0 \mathrm{~s}$ induced due to the interaction phenomenon. In the 1995 Kobe earthquake $(\mathrm{M}=6.9)$ the interaction effect played a vital role in the sudden increase of natural period where the collapse and overturning of Hanshin expressway is observed. In same earthquake, Daikai station failed due to poor load transfer mechanisms due to interface effects [17]. In the 2010 Haiti earthquake $(M=7)$, the collapse of several buildings has observed because of deeper rotation failure due to movement of soils. During the 2001 Bhuj earthquake $(M=7.7)$ caused extensive damage to life and property due to the attenuation effect of the wave travelling through the soil layers with a high impedance contrast of the supporting soil layers. Thus, it is essentially needed to incorporate the flexibility of foundation soil by considering the interaction effect into the calculation [31].

The response of the asymmetrical building investigated by Olariu and Movila [19] by analytical approaches like arithmetic sum method and spectral acceleration method to understand the behavior of shallow foundation by incorporating the interaction effect by spring and dashpot. Mason [18] and Hokmabadi [11] carried out the experimental study with scaled down model of the asymmetrical dwarf building to study the soil structure interaction effect on the structural response under earthquake. Still the approaches not extended for the pile supported asymmetrical buildings. Chopra and Gutierres [5] highlighted out that the numerical methods are most appropriate and accurate methods for soil structure interaction analysis. Followed by this several researchers, including Wegner et al. [29] carried out the study for SSI analysis of the asymmetrical building supported by the isolated, raft and shallow foundation system by considering the 3-D and the 2-D nonlinear analysis. Hadi et al. [10], Tehrani and Khoshnoudian [28] attempted to analyze the nonlinear dynamic SSI system of an asymmetrical building supported by shallow foundation and effect of interaction has been modeled by the spring and dashpot. As the asymmetrical buildings are one of common and unavoidable construction the more attention must be given towards the precise analysis which included the interaction effect. But once the interaction effect included in the numerical analysis the modeling becomes very complex and the time of analysis also increases exponentially due to consideration of soil element and up to the infinite domain.

Thus, it is significantly needed to suggest the approach which simplifies the modeling of SSI system and reduce the time of analysis. This paper aimed to suggest the approach for reducing the complexity in SSI modeling and reducing the analysis time by implementing the Equivalent Pier Method (EPM) for the asymmetrical building supported by piles.

Many methods have been presented in the literature for estimating the settlement of pile foundations, ranging from empirical methods, through simple hand calculation methods, to sophisticated numerical finite element and finite difference analyses. Attempts have been made to trace the development of rational methods of estimating pile group settlements, and the focus has been given to an approach which considers the pile-soil interaction in a proper manner, although it may involve approximations in relation to the modeling of the soil. In all approaches to estimate the group settlement the attention has been concentrated on the relationship between the settlement of a group and that of a single pile. Brief consideration has also been given to the settlement of piled raft foundations, and to the applicability of the simpler methods of analysis. The importance of appropriate estimation of Geotechnical parameters will be emphasized, and finally, it will be demonstrated that misleading results can arise from the imprudent application of group settlement analysis. In this way, an attempt will be made to narrow some of the gaps that have developed between research and practice.

It is now well recognized that the settlement of a pile group can differ significantly from that of a single pile at the same average load level. There are a number of approaches commonly adopted for the estimation of the settlement of pile groups [22]:

- Interaction factor method: It employs the concept of interaction factors and the principle of superposition for the number of piles present in the group.

- The settlement ratio method: It involves the modification of a single pile load settlement curve, to take account of group interaction effects. In this method the settlement of a single pile at the average load level is multiplied by a group settlement ratio, which reflects the effects of group interaction. 
- The equivalent raft method: In this approach the pile group is represented by an equivalent raft acting at some character depth along the piles.

- The equivalent pier method: In this method the pile group is represented by a pier containing the piles and the soil between them. The pier is treated as a single pile of equivalent stiffness in order to compute the average settlement of the group.

The soil pile interaction effect can be implemented with more preciseness in the equivalent pier method than the other empirical methods [21]. Thus, in the present study the dynamic displacement of pile group and the superstructure has been estimated by reducing the pile group into a single pier of equivalent stiffness.

\section{Theory and background of the equivalent pier method (EPM)}

Poulos et al. [22] introduced the method of the equivalent pier method (EPM) to estimate the settlement of the large structure supported on the number of piles. Horikoshi and Randolph employs this method to estimate the overall settlement of piled rafts. In this method number of piles present in the group is replaced by the single equivalent pier [22] as shown in Fig. 1.

In the equivalent pier method (EPM) the pile groups as a whole represented by a single pier to simplify the procedure for estimating the settlement of pile groups. To obtain this, the pile group is replaced by a pier of similar length to the piles in the group and with an equivalent diameter (Deq), estimated as follows [21].

Randolph [24], suggested the diameter of the equivalent pier as follows [21]

$D_{e q}=2 \sqrt{\frac{A_{g}}{\pi}}$ OR 1.13 to $1.27{\sqrt{A_{g}}}$

where, $A_{g}$ is a plan area of pile group, including the soil between the piles.

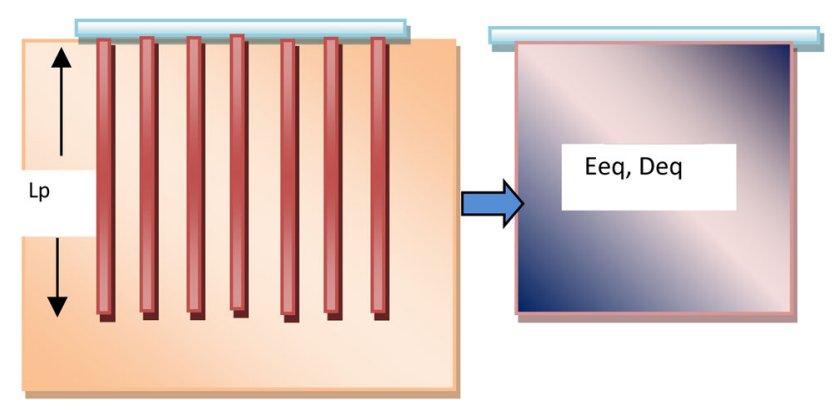

Fig. 1 Concept of the equivalent pier method [21]
The lower value in Eq. 1 is more relevant to end bearing piles, while larger value is more applicable to friction or floating piles.

As in equivalent pier includes the soil entrapped in the pile spacing it is needed to modify the Young's modulus in the analysis. The Young's modulus of the equivalent pier is given by the following equation

$E_{e q}=\frac{\left(E_{P}-E_{S}\right) A_{n p}}{A_{g}}+E_{S}$

where, $E_{p}$ is the Young's modulus of the pile, $E_{s}$ is the Young's modulus of the soil penetrated by the piles, $A_{n p}$ is the total cross sectional area of the piles in a group, $A_{g}$ is the plan area of pile group, including the soil between the piles.

Randolph [24] have examined the accuracy of the equivalent pier method for predicting group settlements, and have concluded that it gives good results [22]. Poulos [21] has examined group settlement as a function of the number of piles, for a group of end bearing piles. Solutions from the computer program DEFPIG, the equivalent raft method and the equivalent pier methods were compared, and for more than about nine piles, the settlements given by all three methods agreed reasonably well. Thus the applicability of EPM has been validated for the symmetric pile group, but there is no attempt has been made for the asymmetrical pile groups. The study extended to understand the effect of pile spacing and length on the settlement of pile group and came up with the suggestions and limitation with respect to the pile aspect ratio coefficient to predict the pile group settlement with more accuracy.

Due to the complexity involved in the soil structure interaction analyses, required for an optimum design, designers have so far been resorting to the traditionally designed pile foundation system permitting very small limiting settlements which violate the safety of the structures. Keeping this in the mind, the researchers like Burland [3] and subsequently Polous [20] had brought out the use of piles with the raft to reduce the settlement of the raft. This had led the advent of the combined piled raft foundation system, which provides a skillful Geotechnical concept to design the foundation for structures which are sensitive to large settlements. The piled raft analysis is a three dimensional interaction problem, wherein, the load transfer mechanism is a complicated interaction process by which the load is shared. The interactive process between the various procedures based on observational studies [15] small scale model studies such as centrifuge models [13] $1 \mathrm{~g}$ model studies [2] and the resulting interactive process with the numerical modeling $[6,25]$ supported by the development of new geotechnical computational facilities [26] has led to the piled raft foundation system being extensively used to support tall and heavily loaded 
structures in a successful manner, permitting larger settlements close to the permissible value $[20,31]$. But still the attempts have been carried out for the symmetric pile group with the interaction effect subjected to the static loads. There is no attempt made to find out the settlement for the asymmetrical pile groups, including the interaction effect subjected to the seismic loading condition. It also have been observed that there is a research gap to understand the behavior of the integrated system, including foundation, soil and the structure by incorporating the interaction effect by reducing the system by the equivalent pier method.

Apart from the suggestion on the theory of the equivalent pier method, [23] focuses on some limitations of the method listed as follows

The method is agreeable for the gravity loading condition induced from the superstructure loading.

The method estimates the vertical strains and settlements in the subsoil.

It assumes that the settlement arises from the consolidation, and that settlement arising from the immediate shear strain is negligible.

The method significantly over predicts the settlement for a relatively small number of piles, but provides a satisfactory solution for 16 or more piles. Conversely, the equivalent pier method tends to unpredicted the settlement as the number of piles in the group increases.

\section{Finite element formulation of soil structure interaction (SSI)}

The generalized equation of motion is used to get the response of the SSI system. When the system is subjected to the earthquake the acceleration, combined with the system mass has been taken as external forces. Equation 3 shows the various components of the SSI system when it is subjected to the ground motion.

$[M]\{\ddot{u}\}+[C]\{\stackrel{\circ}{u}\}+[K]\{u\}=-[M]\left\{\ddot{u}_{g}\right\}$

where, $\mathrm{M}, \mathrm{C}$ and $\mathrm{K}$ are the mass, damping and stiffness matrices of the integrated system which includes the structure and foundation system.

$\ddot{u}, \stackrel{\circ}{u}$ and $u$ are the acceleration, velocity and displacement of the system and $\ddot{u}_{g}$ is the ground motion acceleration applied at the bottom of the soil domain. $\mathrm{C}$ is a damping matrix and given by mass proportionate as given as $[C]=\alpha[M]$ where coefficients $\alpha$ is the damping coefficients.

The solution to Eq. 1 can be given using different methods, including implicit and explicit time integration scheme for a specified time history of ground motion. To obtain the total (absolute) free field acceleration, the bedrock acceleration is added to the acceleration of the top node.

For strong ground motion, the non-linear behavior of the soil should be taken into account in the ground response analysis. For non-linear response analysis, the equation of motion (Eq. 4) is modified as:

$[M]\{\ddot{u}\}+[C]\{\stackrel{\circ}{u}\}+R\{u\}=-[M]\left\{\ddot{u}_{g}\right\}$

in which $R\{u\}$ is the non-linear force-displacement relationship of the system. The nonlinear relationship of the soil is represented by a backbone curve, which represents the variation of shear modulus $(G)$ with the shear strain of the soil. In the present study the nonlinear equation of motion is solved numerically using explicit scheme to obtain the response of the system.

\section{Element formulation}

In the present study, 8-noded brick element and 3-D beam element are used for modeling. The soil, piles and raft are modeled using eight-node brick elements. Each node has three translational degrees of freedom along the $\mathrm{X}, \mathrm{Y}$ and $\mathrm{Z}$ coordinates. The structure part including beams and columns is modeled using 3-D two-node beam elements with six degrees of freedom (three translations and three rotations) at each node. In the present study it has been ensured that the 3D elements are sufficiently small so that the shear locking will not affect the results.

\section{Material model}

There are many material models which define the nonlinear material behavior (Mohr-Coulomb model, Cam Clay model, Drucker Prager model, D-P cap model etc.). But it has been observed that the Drucker-Prager model relatively simple to implement numerically as it describes the state of stress by only three governing parameters like cohesion, friction angle and dilatancy angle which can simulate the nonlinear behavior of the soil under the loads [27]. In the present study the Drucker-Prager material model is used to capture the material nonlinearity. The model uses in the stress space a conical failure surface whose projection in the octahedral plane is a circle and in the meridional plane is a line. The failure surface is the generalization of the of the Mohr-Coulomb failure surface represented by a smooth cone instead of the irregular cone with corners. In classical Drucker-Prager model includes the single yield surface with the associated flow rule without hardening.

The yield condition of the model given by Eq. 5

$F=\alpha I_{1}+\sqrt{J}_{2 D}-k$ 
where,

$\alpha=\frac{2 \times \sin \varnothing}{\sqrt{3} \times(3-\sin \varnothing)}, \quad k=\frac{6 \times C \times \cos \varnothing}{\sqrt{3} \times(3-\sin \varnothing)}$

where $\alpha$ and $k$ are material constants; and the upper left index $t+\Delta t$ (Eq. 7) denotes the end of step.

Stress at the end of time step has been estimated iteratively to update the plastic strain as follows.

${ }^{t+\Delta t} f_{D P}=\alpha^{t+\Delta t} I_{1}+\sqrt{J}^{t+\Delta t} J_{2 D}-k=0$

If the stress estimated at the end of step is not lying on the yield surface the modified stress path has been determined by using a cutting plane algorithm (CPA) [4]

\section{Nonlinear analysis}

Solution of many engineering problems is based on linear approximations. In the actual behavior of the system analyses, these approximations are represented by consideration of small displacements and conservative load conditions. But in some analysis like SSI, where soil behavior is always nonlinear under loads and plays a major role in deviating system response from linear to nonlinear with a considerable deviation, hence it is needed to consider the effect of nonlinearity in the analysis. If these phenomena are included in an FEA, the set of equilibrium equations becomes nonlinear instead of linear. In the present study the SSI system has been solved for static and dynamic load conditions including material nonlinearity. In the present study the implicit time integration scheme is used to carry out static analysis and dynamic analysis has been performed by using explicit time integration method.

\section{Interface element}

The soil structure contact can be modeled by interface element. Several attempts like zero thickness element [9], an isoperimetric virtual element [8], elastic interface element [1], infinite stiffness element [7] have been put forth to understand the contact behavior of the different two bodies in contact after the application of set of load.

The interaction between the pile and the soil surface is inherently non-linear, and has to be solved in the time domain. The equation of motion will be solved using the contact model. In order to solve the problem numerically, the continuous domain is discretized by dividing it in a number of elements. In this study the node to node interface element proposed by Katona [4] has been implemented in order to provide the contact and friction condition between soil and pile. This interface element is capable of responding to tensile separation, frictional sliding or/and complete bonding during the load step.

\section{Node to node contact under static loading condition}

In a static condition a simple friction contact interface element is introduced. This element simulates the frictional slippage, separation and rebounding of two bodies (pile and soil element). The mating of the two nodes in contact at common interface and subsequently deforming with an arbitrary static loading proposed by Katona [14] and Carpenter et al. [4]. Same theory has been implemented numerically in the contact modeling of the interfaces in static loading condition.

\section{Node to node contact under dynamic loading condition}

The Lagrange multiplier method is used to implement the interface state under the dynamic loading in the explicit time integration scheme. In this algorithm at the end of each increment the stiffness matrix need to update based on geometry changes (if applicable) and material changes along with the interface stiffness matrix as explained in static interface implementation procedure. Then a new stiffness matrix is constructed and the next increment of load (or displacement) is applied to the system. In this the response accuracy is obtained in small increments for a large number of time steps. If the number of increments is not sufficient the solution tends to drift from the exact solution. This method does not enforce equilibrium of the internal structure forces with the externally applied loads converges the solution quiet faster than the implicit scheme.

Carpenter et al. [4] suggested the numerical methodology using explicit solvers to get the contact displacement of the nodes in contact (Fig. 2) using the Lagrange multiplier method.

The modified equation of motion when the nodes are in contact using the Lagrange multiplier is given as

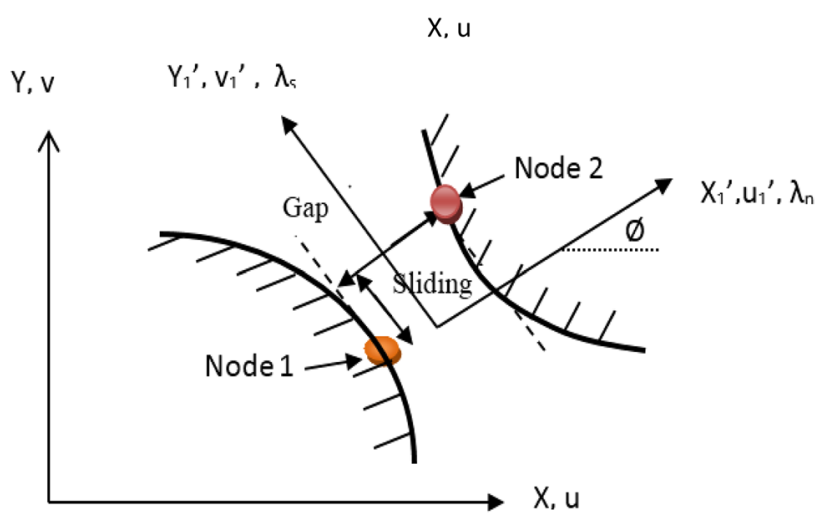

Fig. 2 Schematic representation of node to node interface 


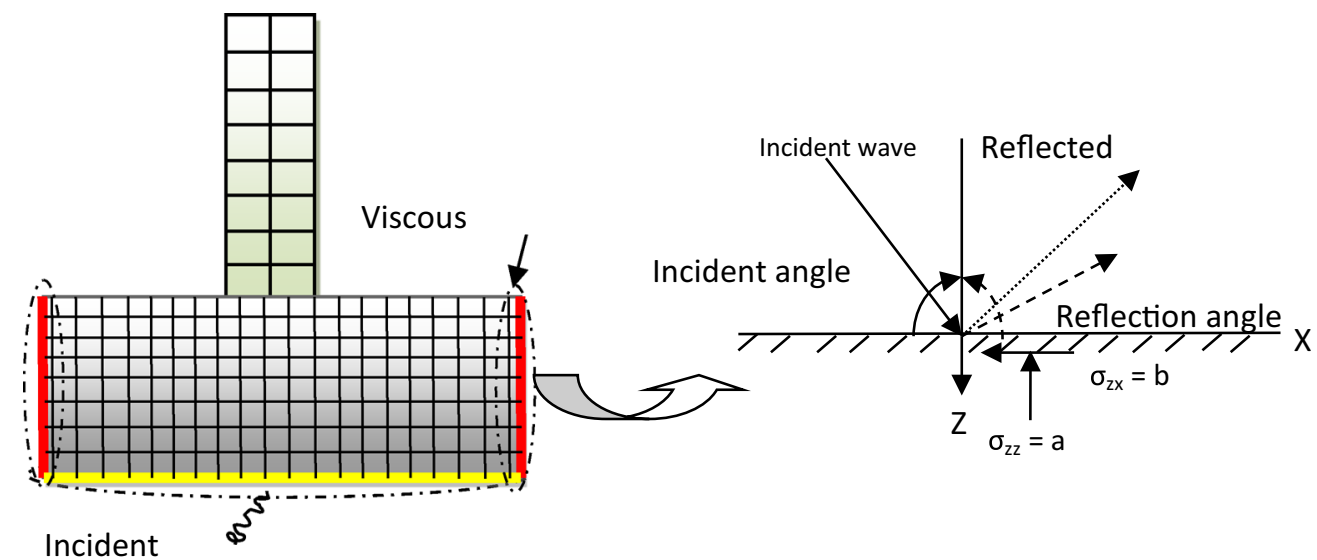

Fig. 3 Schematic representation of a viscous boundary

$M \ddot{u}_{n+1}+f(u, \stackrel{u}{)})+G^{T} \lambda=R$

where, $\lambda$ is the contact forces and $G$ is the contact displacement constraint matrix.

Displacement constraint is prescribed to prevent the node 1 from penetrating the node 2 and to control the tangential sliding of node 2 . The constraint equation in this case can be expressed as

$G\{u+X\}=0$

where, $\mathrm{X}$ is the material coordinate vector of the nodes is contact and $\mathrm{u}$ is the nodal displacement at that time step and sum of $\mathrm{u}$ and $\mathrm{X}$ is the spatial coordinate vector.

Equations 8 and 9 can be written in at $\mathrm{t}_{\mathrm{n}+1}$ th time step as

$M \ddot{u}_{n+1}+K\left(u_{n+1}\right)+G_{n+1}^{T} \lambda_{n+1}=R_{n+1}$

$G_{n+1}\left\{u_{n+1}+x\right\}=0$

Equation 10 can be solved by direct time integration simultaneously to get the penetration vector. The contact displacements are then estimated as given in Eq. 11

$u_{n+1}^{\text {contact }}=-\Delta t^{2} M^{-1} G_{n+1}^{T} \times \lambda_{n}$

The displacement so obtained from the contact forces is then added to the nodal displacement of the respective nodes in contact to get the total response including contact.

\section{Absorbing boundary [16]}

One of the major challenges in dynamic analyses of soilstructure interaction problems is to achieve a balance between accuracy and economic modeling of the far-field medium. Numerous artificial boundaries have been proposed in the literature over the last 30 years. One of the popular techniques is to minimize the theoretically infinite soil domain to avoid the computational expense and can be achieved by introducing artificial boundaries. The great advantage of this approach is that the absorption characteristics are independent of frequency and thus the viscous boundary is suitable for both harmonic and non-harmonic waves. In this study the viscous boundary is implemented in the code.

The energy arriving at the boundary in Fig. 3 will be absorbed if tractions, Eqs. (12), (13) apply to the boundary which is equal in magnitude and opposite in direction to the stresses caused by the incident wave.

$\sigma_{x z}=-b \rho v_{s} \dot{U}_{x}$

$\sigma_{z z}=-a \rho v_{p} \dot{U}_{z}$

The absorption by viscous boundary conditions cannot be made perfect over the whole range of incident angles and/or for all the material properties of the medium, but can be made maximum. Hence, the parameters $a$ and $b$ in Eqs. (12) and (13) can be chosen to maximize the efficiency of the viscous boundary conditions for an arbitrary angle of incidence and material through which waves propagate. A good measure of the ability of the viscous boundary to absorb impinging elastic waves is the energy ratio defined as the ratio between the transmitted energy of the reflected waves and the transmitted energy of the incident wave. This ratio can be computed from the wave amplitude ratios by considering the energy flow to and from a unit area of the boundary. The parameters, $a$ and $\mathrm{b}$ in Eqs. (12) and (13) vary according to not only the incident angles but also the material properties of the medium. The standard or perfectly viscous boundary can be adopted by taking $\mathrm{a}=b=1[16]$. 

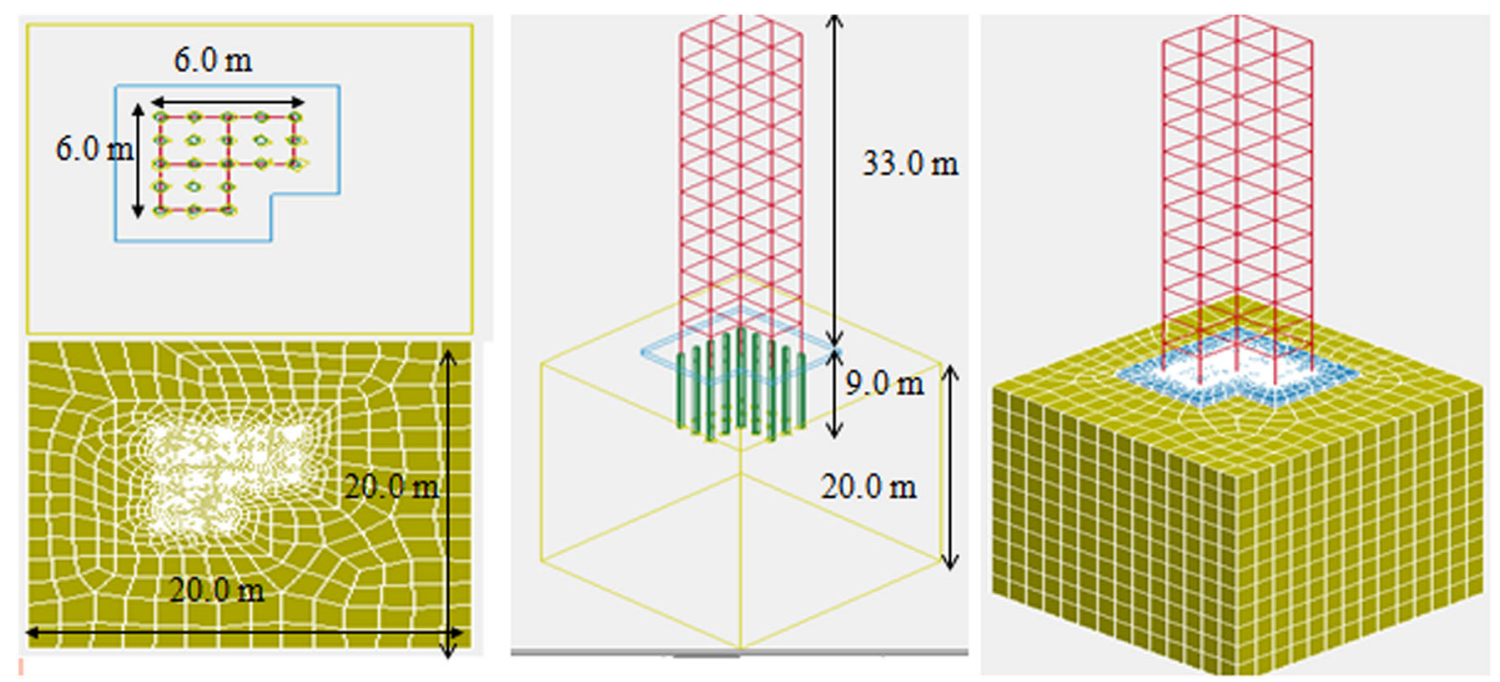

Fig. 4 Soil-pile-structure systems considered in the analysis

\section{Computerization}

A finite element program in $\mathrm{C}++$ has been developed to analyze the SSI system subjected to the earthquake loading. The program can perform nonlinear static and dynamic analysis, including node to node contacts. Following is the vogue to take the input and extract the output from the program

\section{Input}

The input need to be provided through the text files in the specified format with the different files including geometry, i.e. nodes and elements, contact information, boundary conditions, material data, constraints and load data with respect to the DOFs.

\section{File output}

The output file of the program includes the nodal displacement, velocities, accelerations and the elemental stresses. These data from the file has been used to create comparative graphs of the different cases. But in the present document only nodal displacements have been studied to understand the response under the dynamic loading to analyze the applicability of the problem.

\section{Graphical output}

In this study LS-PP is used to show the output graphical views of the SSI system. LS-DYNA-PrePost is the freeware tool for pre and post process which is exclusively used for creating the views/rendering purposes.
The program generates the binary file in a 3D plot format which is the input for LS-PP tool. Binary file includes the data like nodal coordinates, displacement, velocities, accelerations and element stresses at every time step. Thus LS-PP tool has been used effectively for generating building elevation and section.

\section{Modeling the soil-pile-structure system under seismic loading}

In dynamic analysis, the total interaction response is the combination of the two parts namely kinematic and inertial interaction. Wolf [30] has given an understandable shape to the SSI analysis by giving the detailed numerical methods. The soil structure interaction problem can be analyzed using the direct method and substructure methods.

The proposed interaction model is a three-dimensional nonlinear finite element model that consists of L-shape building with the pile foundation subsystems and the reduced model with probable EPM configuration with the suggested approach. In the present study the finite element method (FEM) has been incorporated by developing a program in $\mathrm{C}++$, with object oriented methodology to analyze the interaction effect for pile supported buildings subjected to the transient loading condition. The soil structure interaction problem is very complex and need to model various components like material nonlinearity, an absorbing boundary, interfaces between soil-pile and raftsoil, the connection between superstructure base columnraft and pile-raft etc. The implementation techniques for numerical model of soil structure interaction problem have been explained in details in the preceding sections. The 
Fig. 5 Piled raft layout and details of FE model of the pile. a L-Shape pile layout with raft, b FE model of single pile in a group

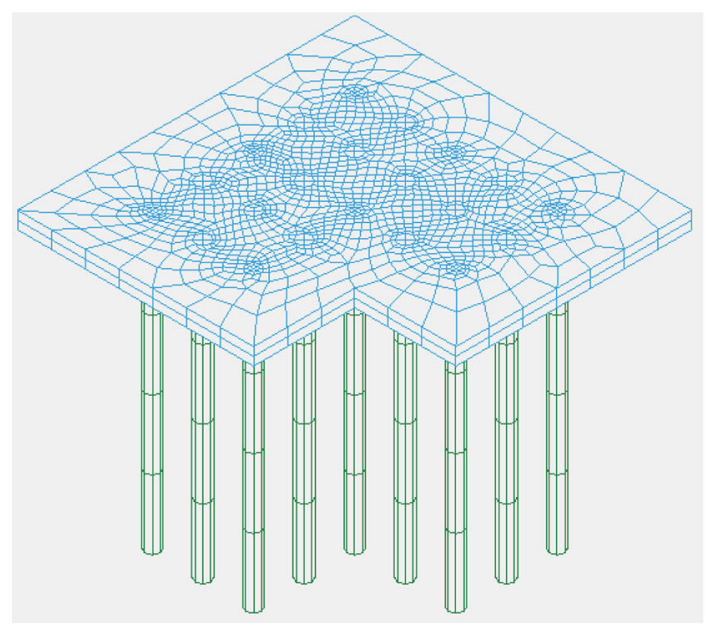

a

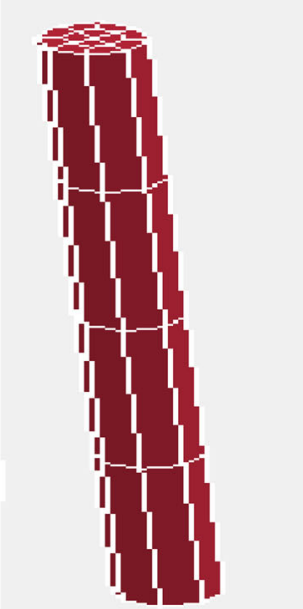

b

Table 1 Details of the data used for modeling

Details of foundation system

\begin{tabular}{|c|c|c|c|c|c|c|c|c|c|}
\hline \multicolumn{2}{|l|}{ Engg. properties } & Unit wt. $\left(\mathrm{kN} / \mathrm{m}^{3}\right)$ & \multicolumn{2}{|c|}{ Friction angle, $\varphi^{\prime}\left(^{\circ}\right)$} & Poisson's Ratio & $\mathrm{E}\left(\mathrm{kN} / \mathrm{m}^{2}\right)$ & Vs $(\mathrm{m} / \mathrm{s})$ & Dar & mping \\
\hline \multicolumn{2}{|l|}{ Soil type: sand } & 18 & \multicolumn{2}{|l|}{35} & 0.35 & 445,872 & 300 & \multicolumn{2}{|c|}{ Mass proportionate } \\
\hline \multirow{2}{*}{\multicolumn{2}{|c|}{$\begin{array}{l}\text { Super structure, pile and raft } \\
\text { Material model parameters for soil }\end{array}$}} & 24 & \multicolumn{2}{|l|}{0} & 0.15 & $2.93 \times 10^{7}$ & 1200 & $\mathrm{Ma}$ & ss proportionate \\
\hline & & \multicolumn{3}{|c|}{ Poisson's ratio $=0.35$} & \multicolumn{2}{|c|}{ Friction angle $=35^{\circ}$} & \multicolumn{3}{|c|}{ Cohesion $\mathrm{C}\left(\mathrm{kN} / \mathrm{m}^{2}\right)=0$} \\
\hline Interface data & & \multicolumn{3}{|c|}{ Friction angle $(\delta)=1 / 3 \phi^{\prime}=11.4^{\circ}$} & \multicolumn{5}{|c|}{ Coefficient of friction $=0.7$} \\
\hline \multicolumn{10}{|c|}{ Details of pile layout } \\
\hline Pile length & Cross section & \multicolumn{2}{|c|}{ Slenderness ratio } & R.C.C Grade & Spacing & \multicolumn{2}{|c|}{ \# piles in layout } & & Layout shape \\
\hline $9.0 \mathrm{~m}$ & 0.45 dia & 20 & & M20 & $1.5 \mathrm{~m} \mathrm{c} / \mathrm{c}$ & 21 & & & L-shape Planer \\
\hline \multicolumn{10}{|c|}{ Details of superstructure } \\
\hline $\begin{array}{l}\text { Bay width and } \\
\text { height }\end{array}$ & \multicolumn{2}{|c|}{$\begin{array}{l}\text { Cross section of floor beam and } \\
\text { columns }\end{array}$} & $\begin{array}{l}\text { Ht. of } \\
\text { building }\end{array}$ & \multicolumn{2}{|c|}{$\begin{array}{l}\text { R.C.C Grade Frame } \\
\text { and rebar }\end{array}$} & $\begin{array}{l}\text { Young's } \\
\text { modulus (E) }\end{array}$ & $\begin{array}{l}\text { Concrete } \\
\text { density }\end{array}$ & & $\begin{array}{l}\text { Asymmetry } \\
\text { shape }\end{array}$ \\
\hline $3.0 \mathrm{~m}$ & \multicolumn{2}{|l|}{$0.23 \times 0.23 \mathrm{~m}$} & $33.0 \mathrm{~m}$ & M20, Fe415 & & $\begin{array}{l}2.0 \times 10^{7} \mathrm{kN} / \\
\mathrm{m}^{2}\end{array}$ & $2400 \mathrm{~kg} / \mathrm{r}$ & & $\begin{array}{l}\text { L-shape } \\
\text { planer }\end{array}$ \\
\hline
\end{tabular}

Fig. 6 Acceleration time history of the 2001 Bhuj ground motion $(\mathrm{PGA}=0.31 \mathrm{~g})$. a Bhuj ground motion, $\mathbf{b}$ part of ground motion considered for study

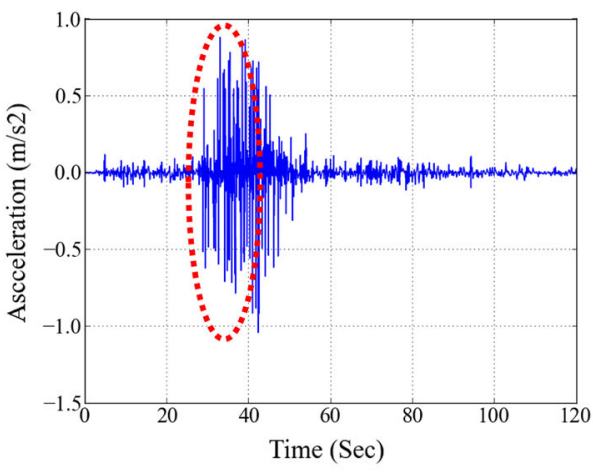

a Bhuj ground motion

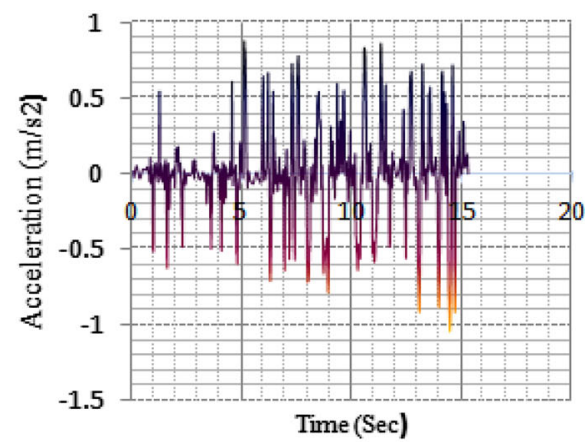

b Part of ground motion considered for study 
Table 2 Details of an equivalent pier for the configurations EPM 1, EPM 2, EPM 3, EPM 4 shown in Fig. 7a-d

\begin{tabular}{|c|c|c|c|c|c|c|c|c|c|}
\hline Area no. & $\mathrm{L}(\mathrm{m})$ & $\mathrm{B}(\mathrm{m})$ & No. of piles participating & $\mathrm{A}_{\mathrm{g}}\left(\mathrm{m}^{2}\right)$ & $\mathrm{D}_{\mathrm{eq}}(\mathrm{m})$ & Es $\left(\mathrm{kN} / \mathrm{m}^{2}\right)$ & $\mathrm{Ep}\left(\mathrm{kN} / \mathrm{m}^{2}\right)$ & $\mathrm{E}_{\mathrm{eq}}\left(\mathrm{kN} / \mathrm{m}^{2}\right)$ & Location $(\mathrm{x}, \mathrm{y})(\mathrm{m}, \mathrm{m})$ \\
\hline \multicolumn{10}{|l|}{ EPM 1} \\
\hline 1 & 3 & 3 & 7.25 & 9 & 3.2 & 445,872 & $2.93 \times 10^{7}$ & $3.93 \times 10^{6}$ & $(1.5,4.5)$ \\
\hline 2 & 3 & 3 & 6.5 & 9 & 3.2 & 445,872 & $2.93 \times 10^{7}$ & $3.41 \times 10^{6}$ & $(1.5,1.5)$ \\
\hline 3 & 3 & 3 & 7.25 & 9 & 3.2 & 445,872 & $2.93 \times 10^{7}$ & $3.93 \times 10^{6}$ & $(4.5,1.5)$ \\
\hline \multicolumn{10}{|l|}{ EPM 2} \\
\hline 1 & 3 & 6 & 13.5 & 18 & 4.5 & 445,872 & $2.93 \times 10^{7}$ & $3.54 \times 10^{6}$ & $(1.5,3.0)$ \\
\hline 2 & 3 & 3 & 7.5 & 9 & 3.2 & 445,872 & $2.93 \times 10^{7}$ & $3.93 \times 10^{6}$ & $(4.5,1.5)$ \\
\hline \multicolumn{10}{|l|}{ EPM 3} \\
\hline 1 & 3 & 3 & 10.5 & 13.5 & 4.2 & 445,872 & $2.93 \times 10^{7}$ & $3.67 \times 10^{6}$ & $(1.67,3.67)$ \\
\hline 2 & 3 & 3 & 10.5 & 13.5 & 4.2 & 445,872 & $2.93 \times 10^{7}$ & $3.67 \times 10^{6}$ & $(3.67,1.67)$ \\
\hline \multicolumn{10}{|l|}{ EPM 4} \\
\hline 1 & 6 & 3 & 21 & 27 & 5.8 & 445,872 & $2.93 \times 10^{7}$ & $3.67 \times 10^{6}$ & $(2.5,2.5)$ \\
\hline
\end{tabular}

Fig. 7 Different trial configurations for equivalent pier considered in the present study
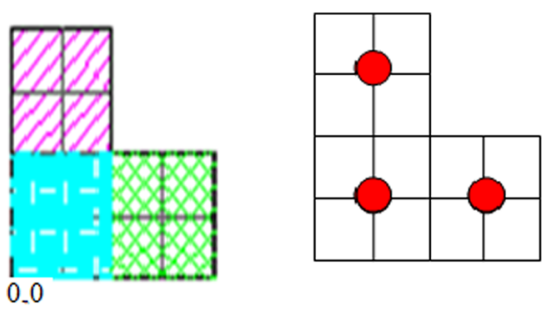

a

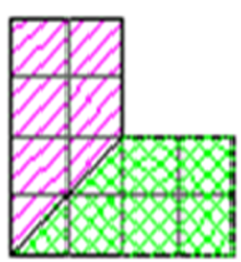

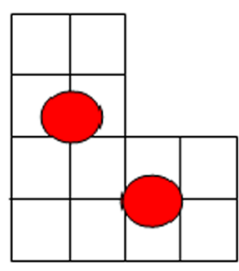
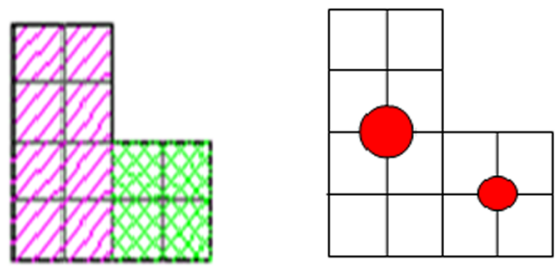

b
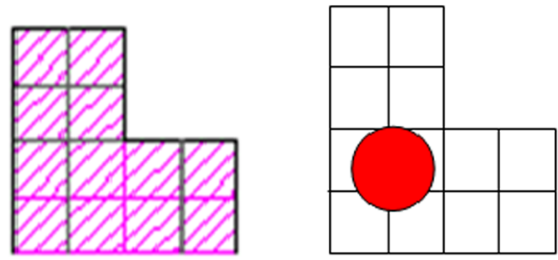

finite element model of the pile soil structure interaction is shown in Fig. 4.

\section{Rocking of the pile group}

For the finite element formulation considered here, eight noded brick elements with three degrees of freedom at each node is used in the analysis. Rotations in the analysis can be represented by three independent displacements (DOFs). Once these displacements are known, three components of normal strains as well as the three components of shear strains (rotations) can be found. In the present analysis, the raft is rigidly connected with pile in all three $\mathrm{x}, \mathrm{y}, \mathrm{z}$ directions. Rocking of the pile can be calculated through the evaluation of rotation of the pile connected to it (rigid body movement of the raft at the piled raft connections). This is a reasonable assumption, as piled raft with dimensions used in practice tend to behave as a rigid body, especially in the lateral direction. The general arrangement of the pile with raft is shown in the Fig. 5a.

\section{Properties of soil pile system}

The properties of the different elements of the model, including soil, pile, raft, structure and interfaces are needed to give as an input in the program developed in the this study to analyze integrated system under earthquake loading. 
Fig. 8 Finite element model for different EPM configuration
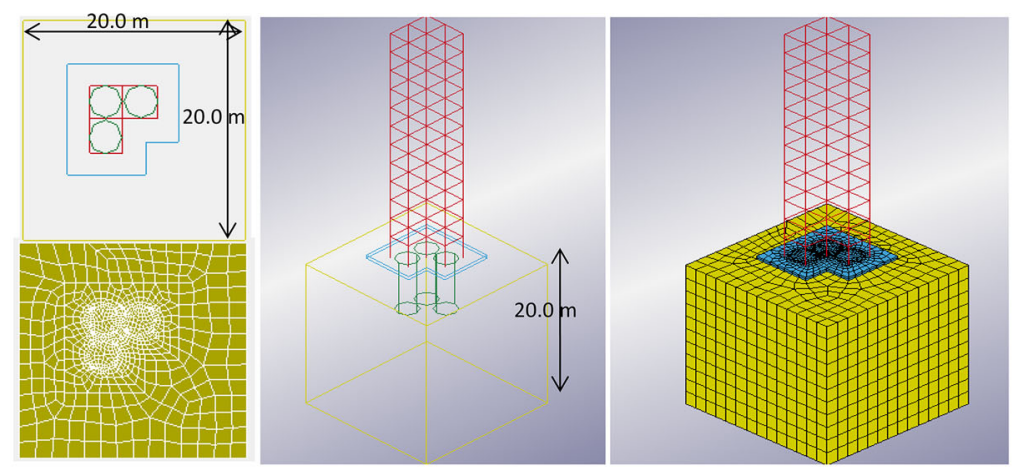

a $\operatorname{EPM} 1$
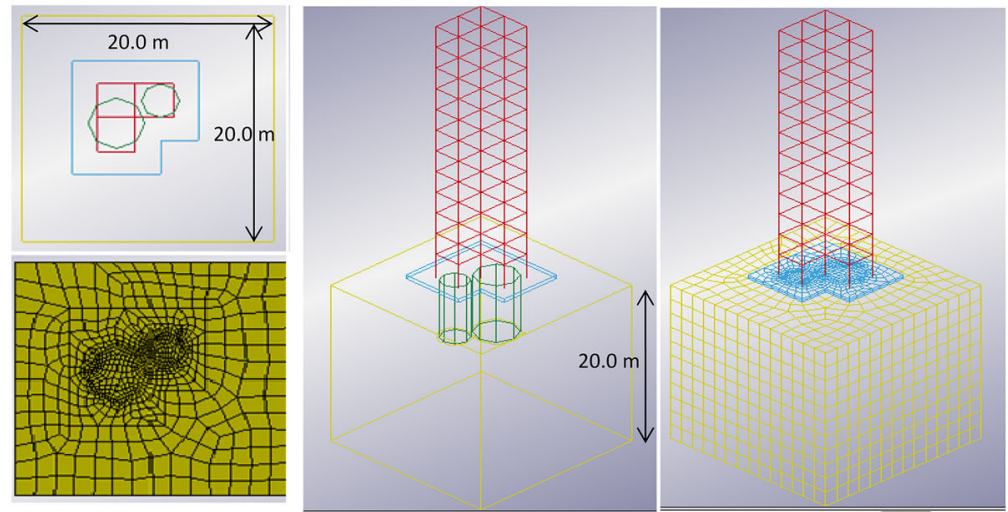

b EPM 2
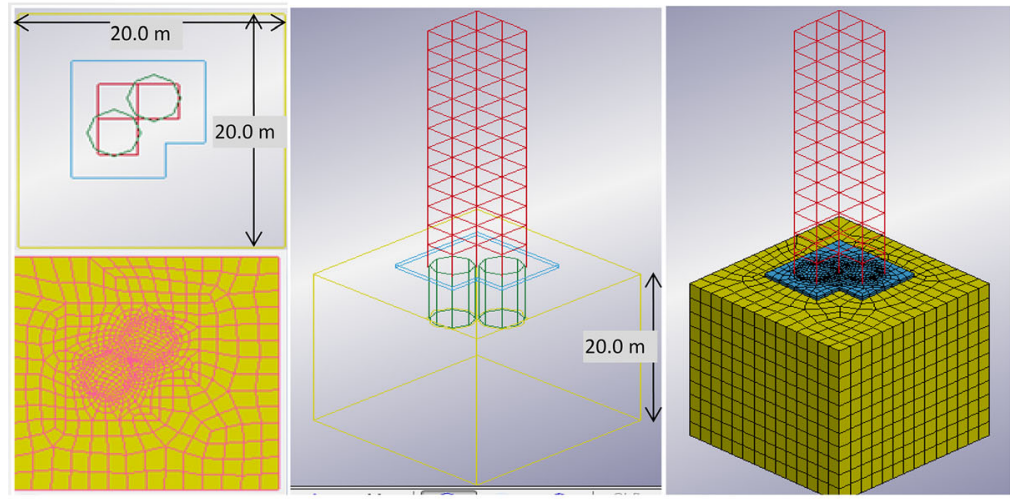

C EPM 3
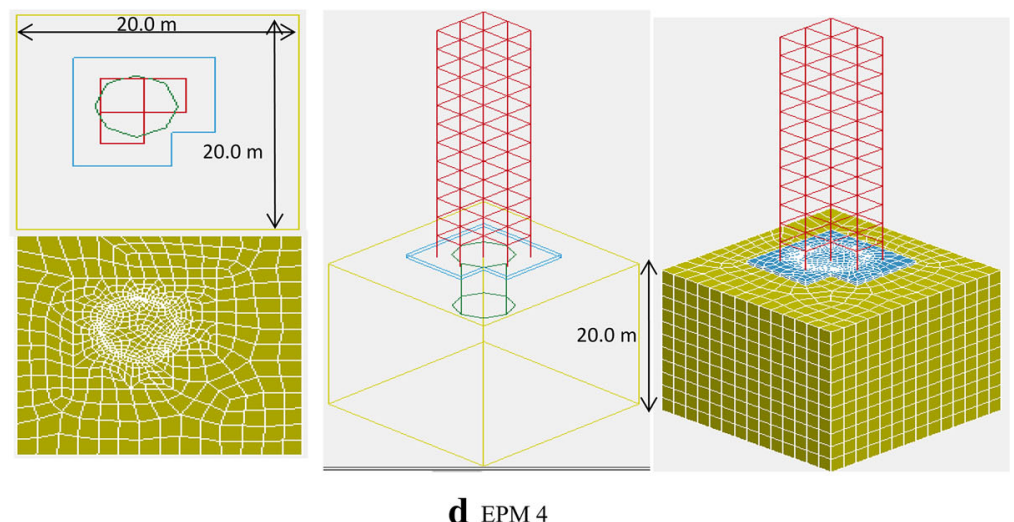


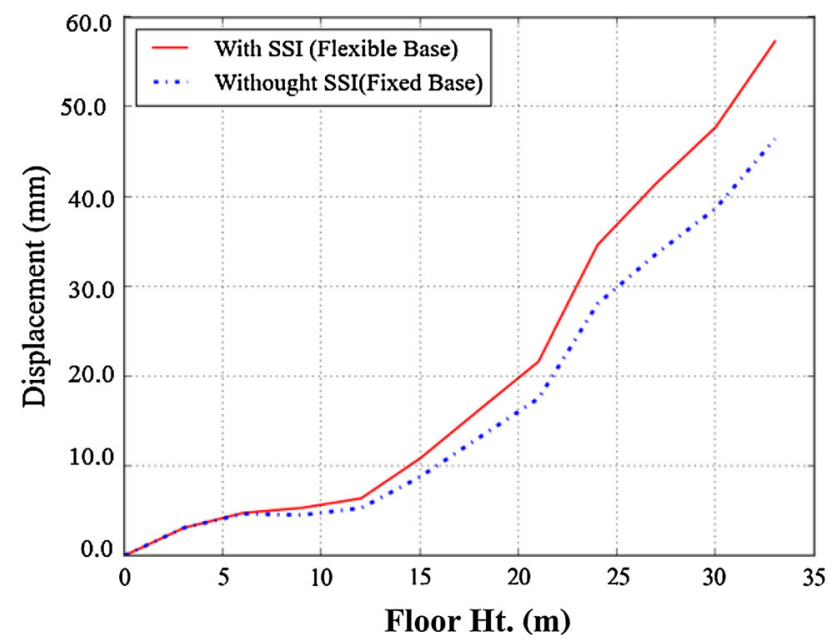

Fig. 9 Floor wise response of asymmetrical building for fixed base and SSI condition in the direction of applied ground motion
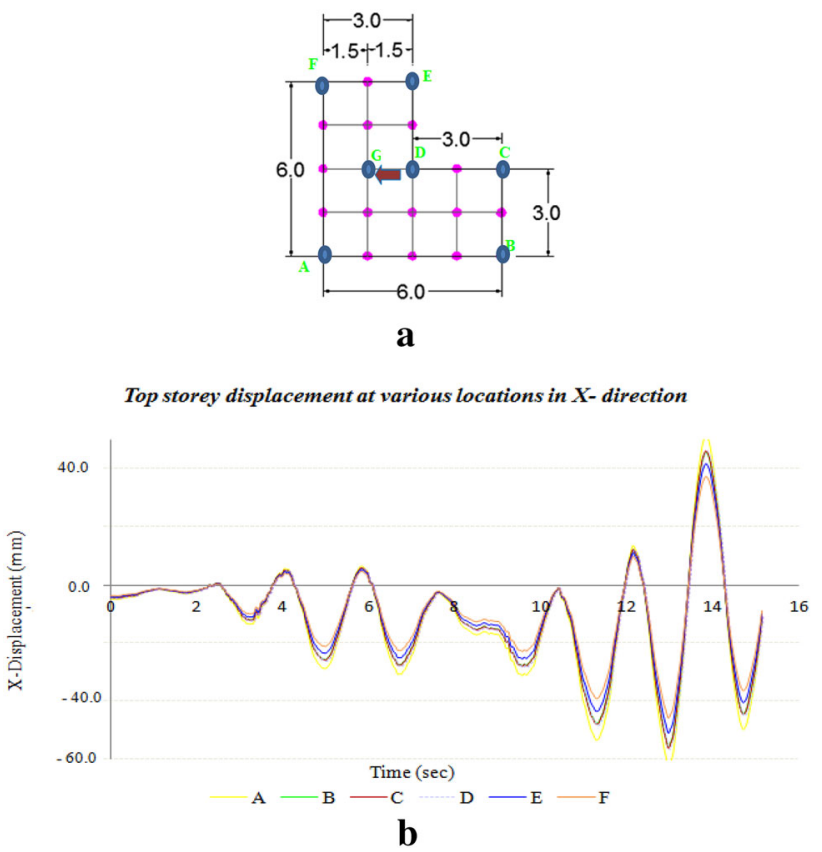

Fig. 10 a Response location of the superstructure. b Top storey response at different locations

The detailed engineering properties of these elements which used to create the finite element model of the system are described in the Table 1.

\section{Earthquake loading}

The stresses and displacement so obtained at the end of static analysis has been considered as the initial response or precondition for the dynamic analysis. The 2001 Bhuj ground motion ( $\mathrm{PGA}=0.31 \mathrm{~g}, \mathrm{E}-\mathrm{W}$ component) has been applied at the bottom nodes of the soil domain and the analysis has been carried out for the peak response which lies in the $15 \mathrm{~s}$ (Fig. 6b). The dynamic responses have been predicted using explicit solver. The material nonlinearity has been considered by adopting the Drucker-Prager material model with associative flow rule and neglecting the hardening effect.

\section{The approach proposed in the present study}

In this study the existing pile group is replaced by the equivalent pier with modified diameter and the modulus of elasticity (Table 2). The method is good enough for the symmetric pile groups, but need to extend and validate its applicability in an asymmetrical pile group with trial and error configuration of the equivalent pier locations (Fig. 7).

\section{Finite element model for EPM configuration}

The finite element model view has been created using LSPP graphical tool by importing the meshed coordinate file in the key format. Following are the FE models (Fig. 8) shown in the different EPM configuration, including EPM 1, EPM 2, EPM 3 and EPM 4. The size of the soil volume, structural configurations and the raft dimensions have been maintained same as the general/existing pile layout.

\section{Analyses for transient excitations}

The soil pile structure system is subjected to the Bhuj (2001, PGA $=0.31 \mathrm{~g}$ ) earthquake and the responses of the pile soil and the structure are estimated. The time history response of the system is estimated for the prescribed ground motion. The effect of soil nonlinearity on the integrated system response is investigated. These consistent observations are made for general pile layout and the various EPM configurations in time domain.

\section{Results and discussion}

The $\mathrm{G}+10$ R.C.C. L-shape asymmetrical building supported by the pile foundation system in a homogenous soil strata is analyzed for Bhuj ground motion (2001, PGA $=0.31 \mathrm{~g}$ ). The results are estimated with the view of the applicability of the Equivalent Pier Method to reduce the computational efforts and the complexity in modeling.

\section{Effect of SSI on building response}

In order to study the effect of SSI the building is analyzed for fixed base condition and flexible base condition, i.e. including the effect of interaction in the analysis. In this 
Fig. 11 Response of superstructure at top storey (at location A)
Top storey response at Location A
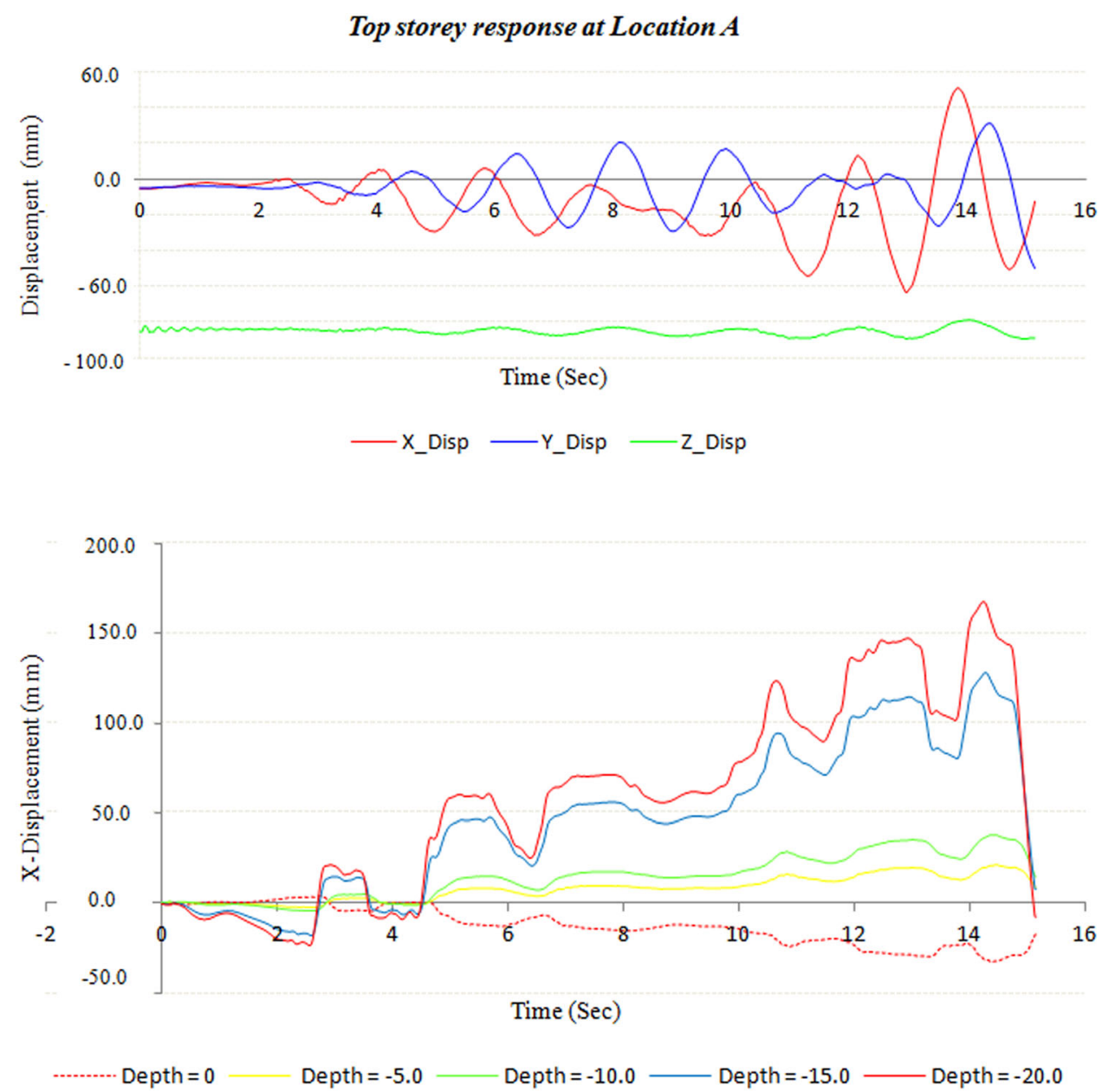

Fig. 12 Soil displacement in $\mathrm{X}$-direction at location $\mathrm{G}$ case the building is subjected to the same dynamic loading with $\mathrm{PGA}=0.31 \mathrm{~g}$ applied at the bottom of the structure and response is calculated in time domain. Figure 9 explains the comparative building peak response for fixed base and flexible base conditions observed at each floor of the superstructure.

\section{Response of the SSI system for general pile layout configuration under earthquake}

The response of the system, including the superstructure and foundation system has been estimated for a general pile layout at the different corners of the building (Fig. 10a). The inertial interaction has been studied by noting responses of the superstructure once the ground motion reaches to the bottom of the structure. The inertial response at each storey height of the superstructure in all corners, including A, B, C, D, E and F in all principal directions $\mathrm{X}$, $\mathrm{Y}$ and $\mathrm{Z}$ have been estimated. Figure $10 \mathrm{~b}$ shows the time history of the displacement at different locations, including all building corners viz. A, B, C, D, E and F at the top storey level of the superstructure in $\mathrm{X}$-direction (in the direction of applied ground motion). The displacements are compared for all the corners of the superstructure. The response comparison at all corners of the superstructure gives the potential idea of the behavior of the superstructure due to asymmetric layout. It has been observed that the displacement at corner $\mathrm{A}$ is more than the other corners and for other corners also the displacements having some deviation from one another.

As it is observed that the point A shows the maximum displacement history than the remaining corners the other principal direction displacements including $\mathrm{Y}$ and $\mathrm{Z}$ also observed for the same point.

Figure 11 shows the time history displacement in $\mathrm{X}, \mathrm{Y}$ and $\mathrm{Z}$ direction at corner $\mathrm{A}$. The displacement profile in $\mathrm{X}$ and $\mathrm{Y}$ direction shows the similar profile with some phase lag. But the $\mathrm{Z}$ direction displacement follows the different profile which is quite gentle as compare to other directions.

In order to understand the effect of kinematic interaction and the wave propagation through the soil mass, the responses have been estimated below the raft in the soil mass. The effect of interaction of soil in the structural part through the pile soil interaction can be understood by studying the 
Fig. 13 Time history of floor wise response of the superstructure at corner A for general pile layout under seismic loading (duration $=15 \mathrm{~s})$. a Time history of displacement in $\mathrm{X}$ direction (gen. pile layout). b Time history of displacement in Y direction (gen. pile layout). c Time history of displacement in $\mathrm{Z}$ direction (gen. pile layout)
X-Displacements at different storey height for general pile layout

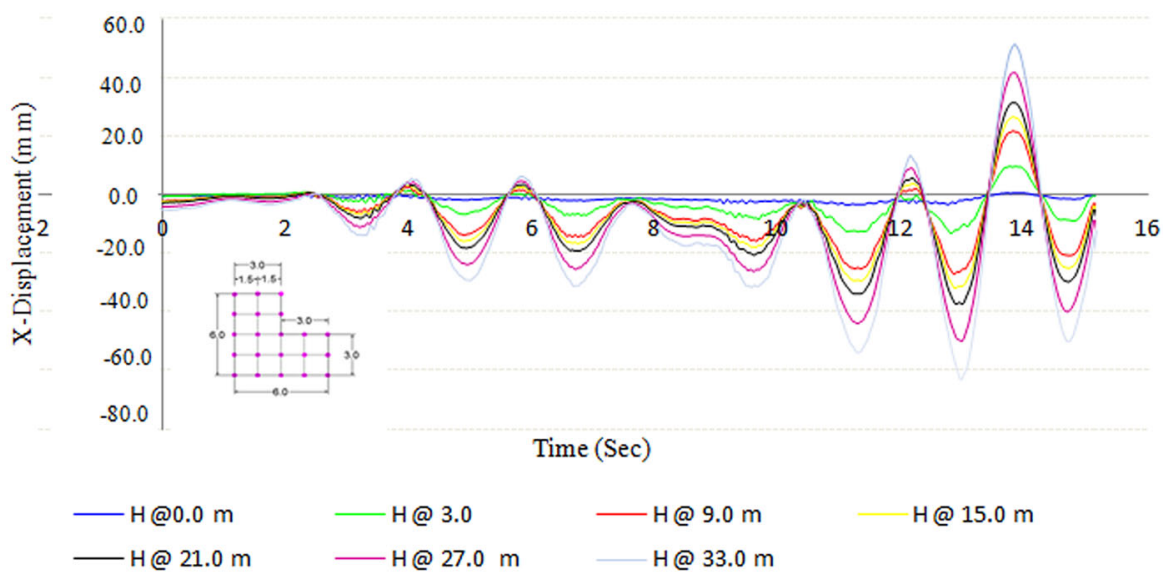

a

Y-Displacement at different Storey height for general pile layout

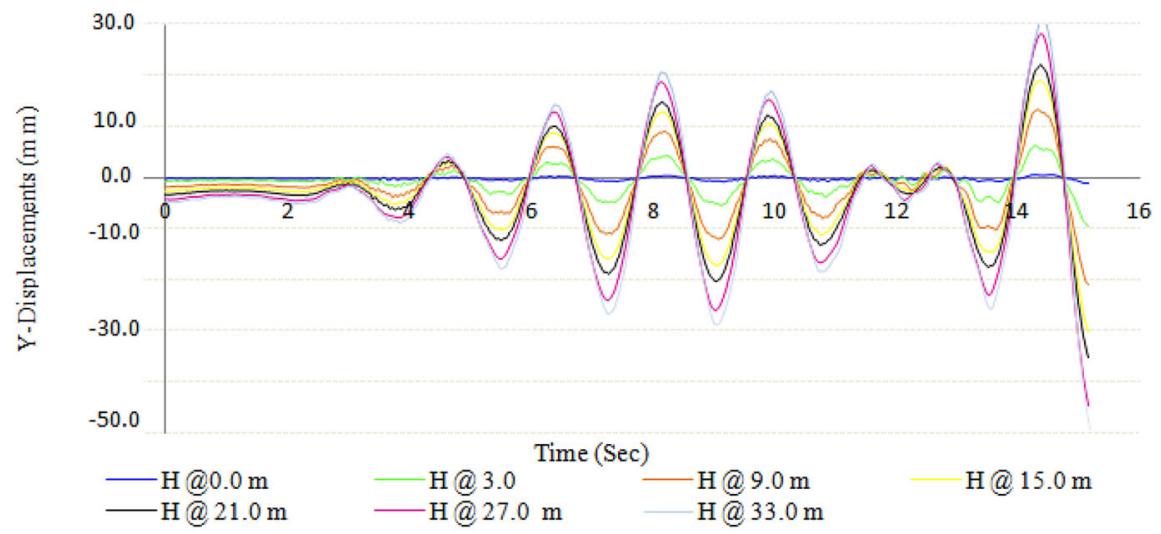

b

Z-Displacement at different Storey height for general pile layout

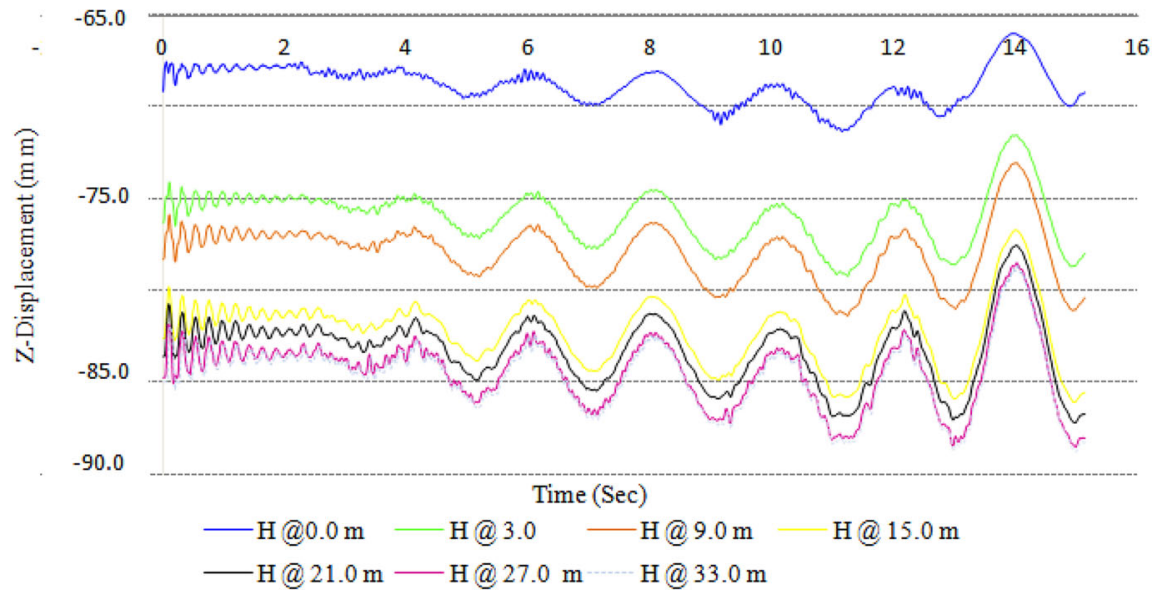


Table 3 Quantitave metric studied in numerical analysis for different configurations considered for the study

\begin{tabular}{lccccccc}
\hline Configuration & DOF s & $\begin{array}{l}\text { Total no. of } \\
\text { elements }\end{array}$ & $\begin{array}{l}\text { No. of } \\
\text { nodes }\end{array}$ & $\begin{array}{l}\text { No. of nodes in } \\
\text { contact }\end{array}$ & $\begin{array}{l}\text { Least element size in FE } \\
\text { model }(\mathrm{m})\end{array}$ & $\begin{array}{l}\text { Critical time step } \\
\text { taken }(\mathrm{s})\end{array}$ & $\begin{array}{l}\text { CPU time to get the } \\
\text { response (h) }\end{array}$ \\
\hline General & 73,927 & 22,515 & 24,665 & 1505 & 0.11 & $5 \times 10^{-5}$ & 51.17 \\
EPM 1 & 39,012 & 11,310 & 13,016 & 495 & 0.15 & $8 \times 10^{-5}$ & 20.10 \\
EPM 2 & 32,275 & 9875 & 11,389 & 397 & 0.15 & $8 \times 10^{-5}$ & 17.50 \\
EPM 3 & 32,725 & 10,015 & 11,539 & 407 & 0.15 & $8 \times 10^{-5}$ & 18.05 \\
EPM 4 & 30,653 & 8872 & 10,227 & 328 & 0.15 & $8 \times 10^{-5}$ & 15.35 \\
\hline
\end{tabular}

time history displacement profile of the different points along the various depths. Figure 12 shows the displacement at location $\mathrm{G}$ below the raft level till the end of soil domain i.e. $20 \mathrm{~m}$ depth. The displacements are observed in the $\mathrm{X}, \mathrm{Y}$ and $\mathrm{Z}$ directions. The input ground motion has been given at the depth of $20.0 \mathrm{~m}$ in $\mathrm{X}$ direction. It is observed that the displacement history at $20.0 \mathrm{~m}$ depth is more compared to the other depths. The displacement at the ground level (level $0.0 \mathrm{~m}$ ) is observed to be least.

At each storey the displacements are estimated to understand the behavior of superstructure under the seismic loading. Figure 13 shows the structural response in $\mathrm{X}, \mathrm{Y}$ and $\mathrm{Z}$ direction for the general pile layout which consists of 21 no. of pile in L-shape layout spaced at $1.5 \mathrm{~m} \mathrm{c} / \mathrm{c}$ with the slenderness ratio 20 .

\section{Response comparison of the SSI system for different EPM configuration under earthquake}

In the present study, the existing pile group is replaced by the equivalent pier with modified diameter and the modulus of elasticity (Table 3 ) in order to check the applicability of the method for the asymmetrical pile groups. The comprehensive analysis has been carried out for the different sets of EPM configuration of the equivalent pier located on the trial basis. The applicability of the equivalent pier method is checked by studying the response of the superstructure by replacing the existing piles by an equivalent pier/s depending upon the area in which the asymmetrical building divided. In this way the 4 trials named EPM 1, EPM 2, EPM 3 and EPM 4 have been figured out and for each trial the building response is estimated.

The CPU time required to obtain the converged nonlinear dynamic solution has been noted for each EPM configuration to check the numerical expense comprises with each mode derived from the different EPM configuration. Figure 14 shows the comparative responses of superstructure obtained for various EPM configurations and the general pile layout, obtained at the bottom $(\mathrm{H}=3.0 \mathrm{~m})$ and top of the $(\mathrm{H}=33.0 \mathrm{~m})$ superstructure.
The numerical adaptability of the proposed approach for soil structure interaction of the pile supported building has been checked by measuring the CPU running time to get the converged solution. The Table 3 shows the quantitative metric for each EPM configuration to understand the numerical expense comprise with each model in details.

In order to have a quick review of responses the peak responses at each superstructure floor compared for different EPM and general pile configuration. Table 4

The deviation in the response of each EPM configuration w.r.t. the general pile layout has been estimated to understand the behavior of the system and its suitability in the approach proposed in the study. Table 5 explains the percentage deviation from general pile layout comprises with each configuration.

\section{Effect of soil types input and ground motion}

The behavior of the Geotechnical material plays important role in changing the characteristics of the seismic waves. Such behavior is especially important in earthquake engineering for defining the dynamic response of soil deposits for the purpose of earthquake resistant design (Buchen 1971). Typically, when soil structure interaction refers the kinematic interaction wholly depends upon the type of soil in turns of its engineering properties and state parameters. Thus, it is at most important to understand the seismic response of the superstructure for various soil types and its state parameters. With concern to this, in the present study different soil type considered are, cohesionless soil (sandy soil, S1), C-Phi soil (sandy clay, S2) and cohesive soil (clay, S3). Table 6 gives the detailed idea of the engineering properties of the soil considered.

The responses at the top of the superstructure have been studied for the different soil types. The comparative study has been made to understand the effect of soil type on the superstructure response (Fig. 15). 
Fig. 14 Response comparison at different height of building for various EPM configurations. a Displacement history in $\mathrm{X}$ direction at ground floor. b Displacement history in $\mathrm{Y}$ direction at ground floor. c Displacement history in Z direction at ground floor. d Displacement history in $\mathrm{X}$ direction at top floor.

e Displacement history in $\mathrm{Y}$ direction at top floor.

f Displacement history in $\mathrm{X}$ direction at top floor
X-Drection response @ $\mathrm{H}=\mathbf{3 . 0} \mathrm{m}$

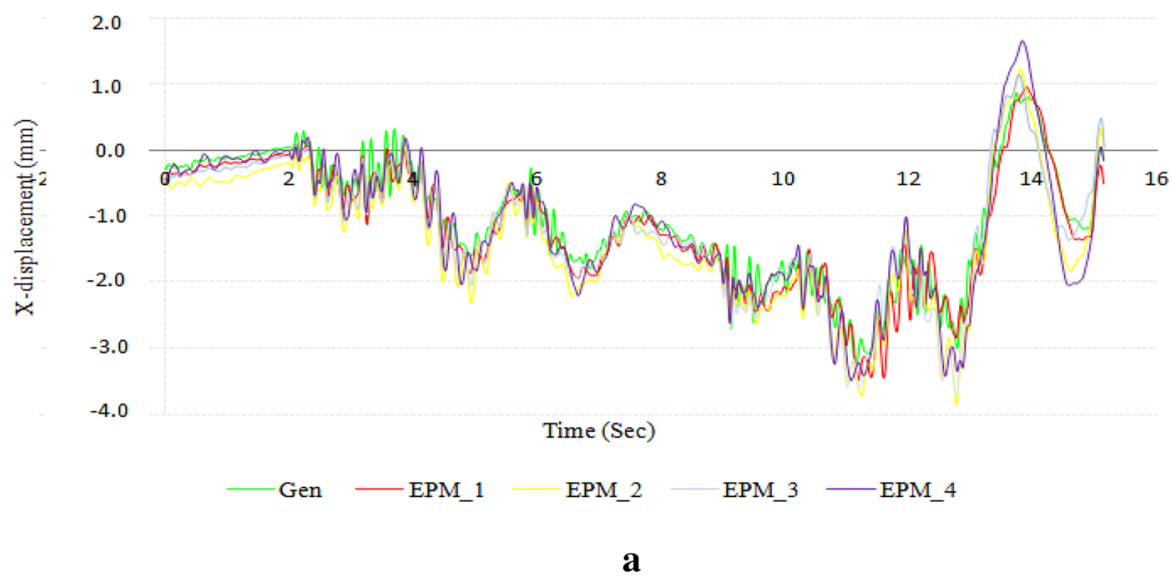

Y-Drection response @ $\mathbf{H}=\mathbf{3 . 0} \mathrm{m}$

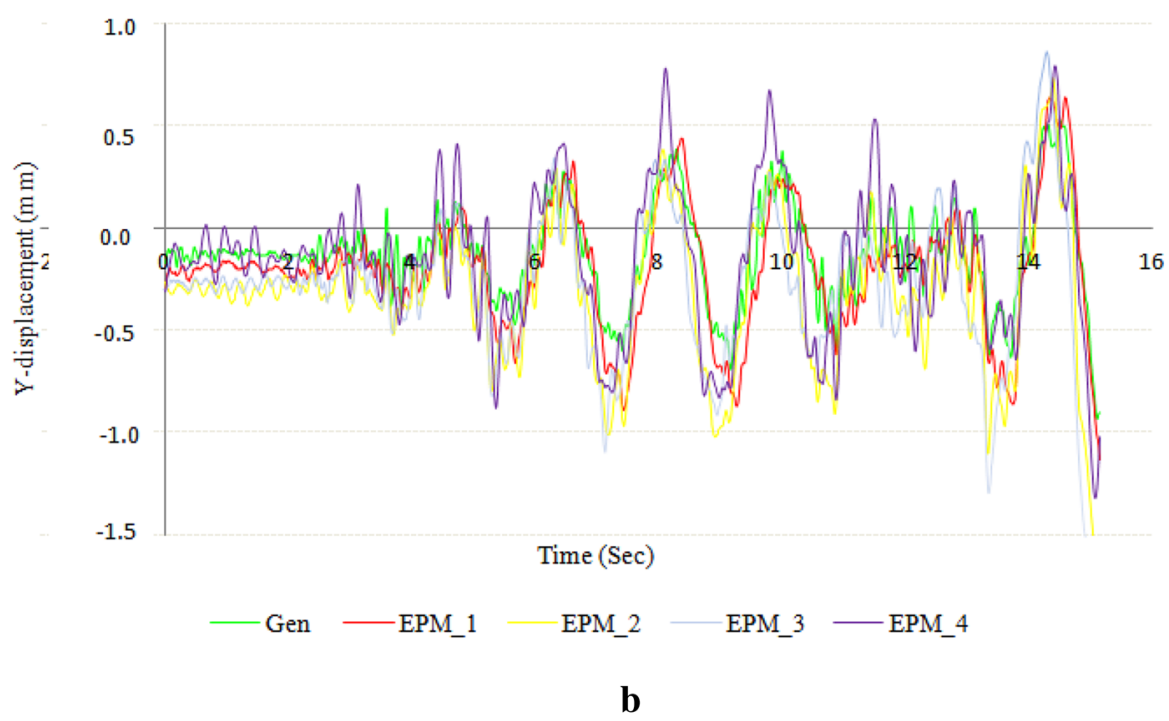

Z-Drection response @ $\mathrm{H}=\mathbf{3 . 0} \mathrm{m}$

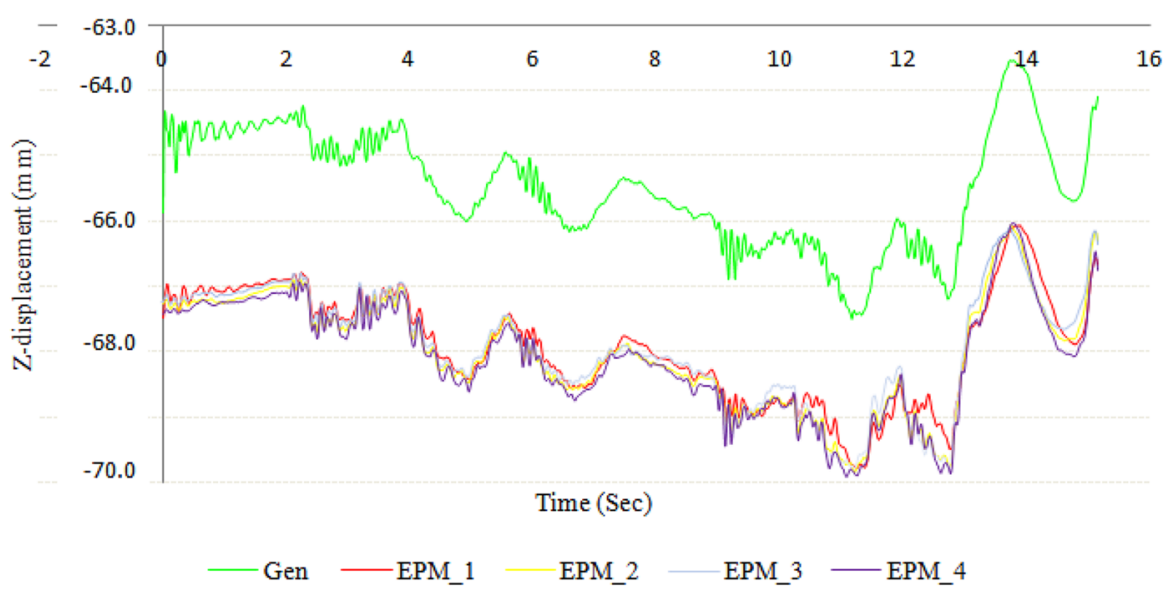

c 
Fig. 14 continued

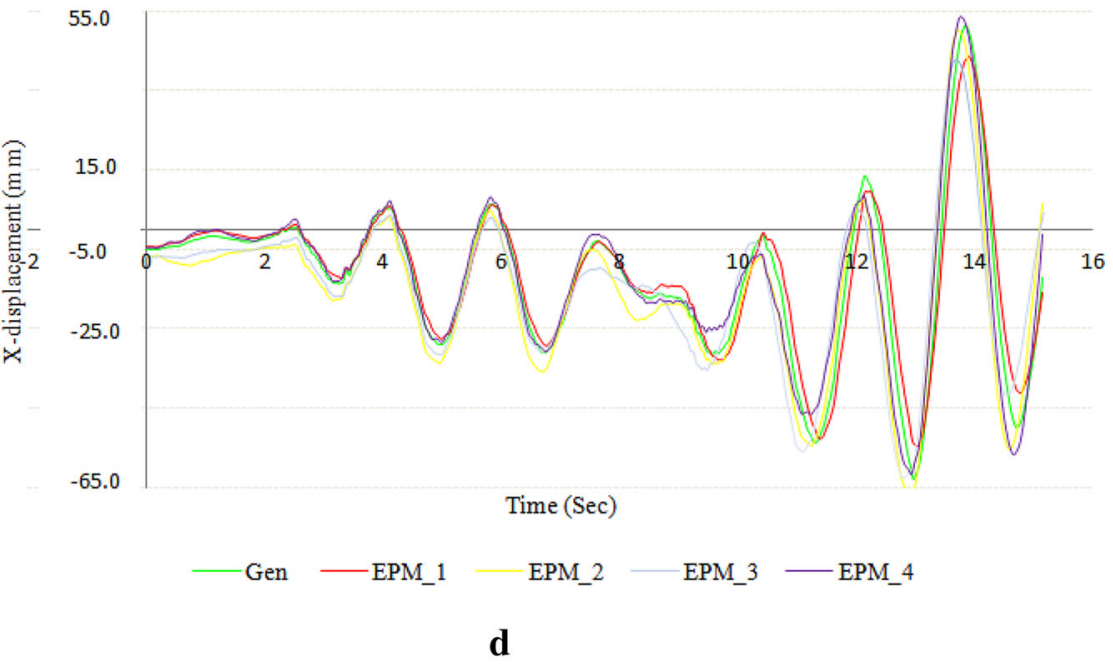

Y-Drection response @ $\mathbf{H = 3 3 . 0 ~ m ~}$
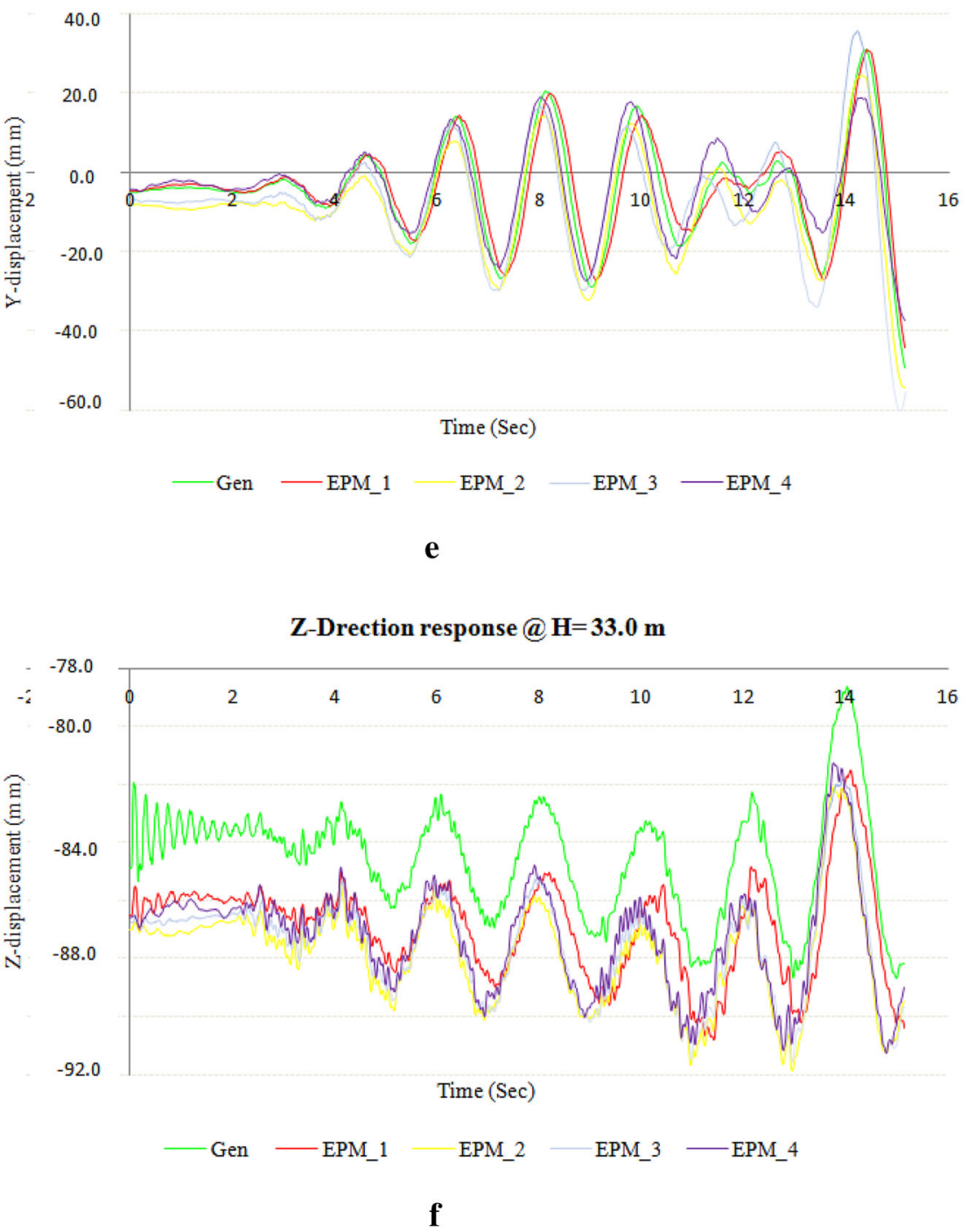
Table 4 Peak response at the top of superstructure for different EPM configuration

\begin{tabular}{llllll}
\hline Configuration & Gen & EPM 1 & EPM 2 & EPM 3 & EPM 4 \\
\hline X-disp. (cm) & 6.29 & 5.46 & 6.74 & 6.26 & 6.16 \\
Y-disp. (cm) & 4.91 & 4.39 & 5.41 & 5.96 & 3.72 \\
Z-disp. (cm) & 8.87 & 9.19 & 9.13 & 9.15 & 9.13 \\
\hline
\end{tabular}

Table 5 Percentage deviation in response for various EPM

\begin{tabular}{lrrrr}
\hline Configuration & EPM 1 & EPM 2 & EPM 3 & EPM 4 \\
\hline X-disp. (\%) & -13.20 & 8.24 & -0.45 & -2.08 \\
Y-disp. (\%) & -10.59 & 11.39 & 19.41 & -19.97 \\
Z-disp. (\%) & 2.37 & 3.52 & 3.05 & 2.84 \\
\hline
\end{tabular}

The behavior of the system is under different dynamic loading has been studied by applying the different ground motion at the bottom of the soil domain. The various ground motions considered for the study includes, the 1995 Chamba $(M=4.9)$, the 1999 Uttarkashi $(M=6.9)$, and the 2001 Bhuj $(M=7.7)$. The specific details of each earthquake have been provided in Table 7 .

The dynamic time history analysis for different ground motion has been carried out and the peak displacement profile of the top storey has been studied. Deviation in peak responses for each ground motion for the various model configurations, including General pile layout and EPM 1, EPM 2, EPM 3 and EPM 4 has been studied for the each applied ground motion. Figure 15 shows the histogram of peak responses for various ground motion.

\section{Conclusions}

The study has drawn the following salient conclusion from the results observed.

It has been clearly noticed that the effect of introducing interaction effect deviates the system response. The deviation so observed is less for the bottom floor and increases as the storey height more. The response is found to be more about $15-20 \%$ on average in case of SSI analysis than the fixed base analysis. Thus, it signifies that the effect of interaction plays important role in superstructure response.

It has been found that, the asymmetrical building response at all points located at the same level is not same and clearly shows that the asymmetrical building has different movement at different location on the same vertical level. This deviation is found to be within the range of $2-4 \%$ for different corners which indicates the effect the structure.

Overall all EPM configurations give the response with the maximum deviation of $+20,-20 \%$. It has been observed that considering the responses collectively in all directions EPM configuration at one of the agreeable approaches to model the L shape pile layout. Thus the approach proves to be good for reducing the SSI model size in terms of interface nodes and total number of elements.

It has been observed that in EPM mechanism, it is needed to model reduced number of the piers depending upon the asymmetrical area involves in the plan geometry of the superstructure, the modeling complexity reduced at the countable extent as the number of piles model at the different locations get reduced at the countable extent.

In this study the solution has been obtained by the explicit solver where the time step is needed to be taken very small and depends upon the least element size in the finite element model. In EPM approach the equivalent piers gives the larger diameter, which gives the bigger size elements after meshing. This facilitates to take the larger time step which is one of the prime factors which reduces the solution time by $68 \%$ (average). Thus the approach so proposed in present study facilitates to reduce the analysis time of the complex system like soil structure interaction. Hence the EPM approach extended to the asymmetrical pile groups is satisfactory for the SSI problems where the numerical cost and CPU memory is required very high.

The floor wise peak displacement values are found to be more in case of cohesion less soil as a supporting stratum than the other two types of soil C- $\varnothing$ and cohesive soils. It has been noted that the difference in the peak displacement

Table 6 Engineering properties of the soil considered for parametric study

\begin{tabular}{lllllllr}
\hline Sr. no. & Soil type & $\begin{array}{l}\text { Young's } \\
\text { modulus } \\
\left(\mathrm{kN} / \mathrm{m}^{2}\right)\end{array}$ & $\begin{array}{l}\text { Poisson's } \\
\text { ratio }\end{array}$ & $\begin{array}{l}\text { Density } \\
\left(\mathrm{kN} / \mathrm{m}^{3}\right)\end{array}$ & $\begin{array}{l}\text { Shear } \\
\text { wave } \\
\text { velocity } \\
(\mathrm{m} / \mathrm{s})\end{array}$ & $\begin{array}{l}\text { Angle of } \\
\text { friction } \\
\left({ }^{\circ}\right)\end{array}$ \\
\hline 1 & $\mathrm{~S} 1$ & 645,872 & 0.30 & 20 & 300 & 42 & 0 \\
2 & $\mathrm{~S} 2$ & 545,872 & 0.30 & 20 & 200 & 30 & 20 \\
3 & $\mathrm{~S} 3$ & 445,872 & 0.35 & 18 & 100 & 0 & 30 \\
\hline
\end{tabular}


Fig. 15 Response comparison for different parameters for various EPM configurations
Effect of soil type and eartquake input on different model configuration

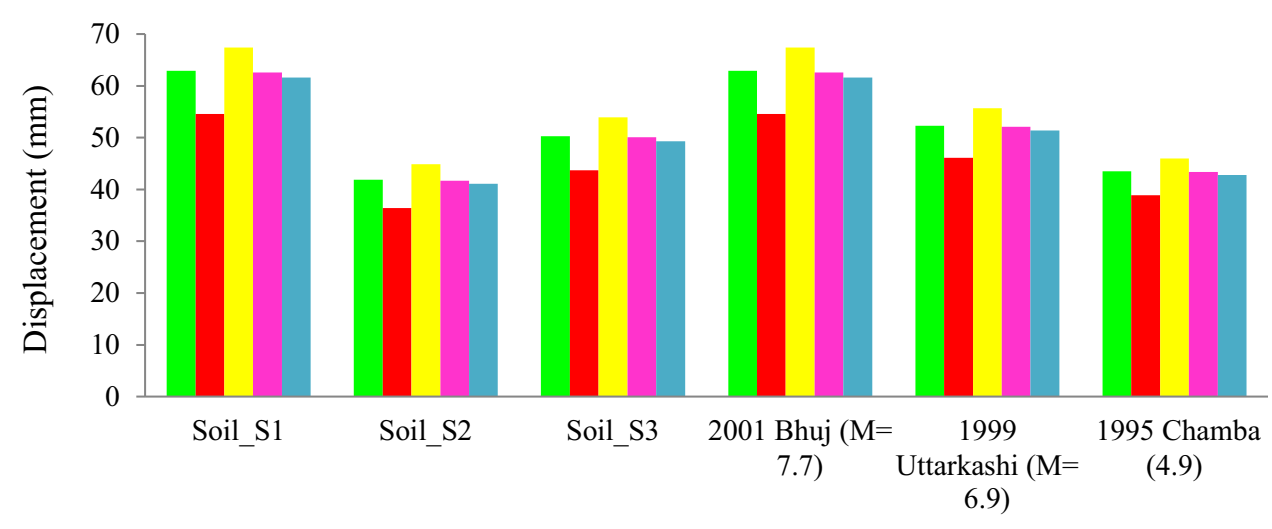

Parameters

a Gen $\square$ EPM 1 EPM 2 EPM $3 \backsim$ EPM 4
Table 7 Details of earthquake considered for the present study

\begin{tabular}{llll}
\hline Earthquake name & Magnitude & PGA $\left(\mathrm{m} / \mathrm{s}^{2}\right)$ & Predominant frequency $(\mathrm{Hz})$ \\
\hline Mar 24, 1995 Chamba (NE) & 4.9 & 1.24 & $0.35-3.53$ \\
Mar 29, 1999 Uttarkashi (NW) & 6.9 & 2.48 & $0.92-4.24$ \\
Jan 26, 2001 Bhuj (NE) & 7.7 & 3.01 & $1.32-4.40$ \\
\hline
\end{tabular}

values lies in the range of $12-15 \%$. And same deviation has been observed in case of other EPM configurations. Thus, the EPM model which makes the SSI model computationally more efficient shows the same variation in responses as observed in the general pile layout model. Thus it shows that the EPM technique can be well adapted for all types of soil.

In this study, when the system analyses for different earthquakes ranging the magnitude from 4.9 to 7.7. The response of the system is observed to be greater for the Bhuj ground motion with peak response greater by $35-40 \%$ than the Chamba ground motion. This difference is around $25 \%$ less for the Uttarkashi earthquake than the Bhuj ground motion. Thus, the study concluded that for high magnitude earthquake kinematic interaction enhances and the effect observes on the superstructure is more than the medium and small earthquake magnitude. Thus the magnitude of the earthquake play very crucial role in the kinematic interaction in SSI analysis and EPM techniques can well be adopted for mild to higher magnitude earthquake.

Open Access This article is distributed under the terms of the Creative Commons Attribution 4.0 International License (http://crea tivecommons.org/licenses/by/4.0/), which permits unrestricted use, distribution, and reproduction in any medium, provided you give appropriate credit to the original author(s) and the source, provide a link to the Creative Commons license, and indicate if changes were made.

\section{References}

1. Alonso EE, Carol I (1985) Foundation analysis of an arch dam, comparison of two modeling techniques: no tension and jointed rock material. J Rock Mech Rock Eng 18:149-182

2. Balakumar V (2008) experimental studies of model piled raft on sand and field study of prototype behavior. Dissertation, Anna University Chennai

3. Burland JB (1995) Piles as settlement reducer. In: Abstract of 18th Italian congress on soil mechanics, Pavia

4. Carpenter NJ, Taylor RL, Katona MG (1990) Surface contact in finite element method. Wiley, New York

5. Chopra AK, Gutierres JA (1973) Earthquake analysis of multi storey buildings including foundation interaction. Report no. EERC73-13, University of California, Berkeley

6. Clancy P (1993) Numerical analysis of piled raft foundations. Dissertation, University of Western Australia

7. Desai CS, Lightner JG (1985) Mixed finite element procedure for soil-structure interaction and construction sequences. Int J Numer Methods Eng 21:801-824

8. Desai CS, Phan HV, Perumpral JV (1982) Mechanics of threedimensional soil-structure interaction. J Eng Mech ASCE 108(5):731-747

9. Goodman RE, Taylor RL, Brekke TL (1968) A model for the mechanics of jointed rock. Int J Soil Mech Found Eng ASCE 94:637-659

10. Hadi MNS, Uz ME (2011) Seismic history analysis of asymmetrical adjacent buildings with soil-structure interaction consideration. In: 8th World conference on earthquake resistant engineering structures. WIT Press, Wessex, pp 225-236

11. Hokmabadi AS, Fatahi B, Samali B (2014) Assessment of soilpile-structure interaction influencing seismic response of midrise buildings sitting on floating pile foundations. Comput Geotech 55:172-186 
12. Hora M (2006) Nonlinear interaction analysis of infilled building frame-soil system. J Struct Eng 33(4):309-318

13. Horikoshi K (1995) Optimum design of piled raft foundations. Dissertation, University of Western Australia

14. Katona MG (1983) A simple contract friction interface element with applications to buried culverts. Int J Num Anal Meth Geomech 7:371-384

15. Katzenbach R, Arslan V, Moorman C H (2000) Numerical stimulations of combined piled raft foundations for the new high rise building. In: Proceedings of the 2nd international conference on soil structure interaction in urban civil engineering, Frankfurt

16. Lysmer J, Kuhlemeyer RL (1969) Finite dynamic model for infinite media. J Eng Mech (ASCE) 95:859-877

17. Maheshwari BK, Trumana KZ, El Naggar MH, Goulda PL (2004) Three-dimensional nonlinear analysis for seismic soil-pilestructure interaction. Soil Dyn Earthq Eng 24:343-356

18. Mason HB, Trombetta NW, Chen Z, Bray JD, Hutchinson TC, Kutter BL (2013) Seismic soil-foundation-structure interaction observed in geotechnical centrifuge experiments. Soil Dyn Earthq Eng 48:162-174

19. Olariu CP, Movila M (2014) Spectral analysis for an asymmetrical structure considering soil structure interaction. Dissertation, Technical University Canada

20. Polous HG (2008) The piled raft foundation for the Burj Dubaidesign and performance IGS-Ferroco, Terzaghi, Oration

21. Poulos HG (1993) Settlement prediction for bored pile groups, Deep foundations on bored and auger piles, A.A. Balkema, Rotterdam, 103-117
22. Poulos HG, Davis EH (1990) Pile foundation analysis and design. Krieger Publication, Malabar

23. Poulos HG, Davis EH (1980) Pile foundations analysis and design, 1st edn. Wiley, New York

24. Randolph MF (1994) Design methods for pile groups and piled rafts, Thirteenth International Conference on Soil Mechanics and Foundation Engineering, India, 5:61-82

25. Russo G (1998) Numerical analyses of piled rafts. Inte J Numer Anal Methods Geomech 22:477-493

26. Small J, Poulos HG (2007) A method of analysis of piled raft. In: Abstract 10th Australia New Zealand conference on geo mechanics, p 555

27. Tahghighi H, Konagai $K$ (2007) Numerical analysis of nonlinear soil-pile group interaction under lateral loads, Soil Dyn Earthq Eng 27(5):463-474

28. Tehrani MH, Khoshnoudian F (2014) Extended consecutive modal pushover procedure for estimating seismic responses of one-way asymmetric plan tall buildings considering soil-structure interaction. Earthq Eng Eng Vib 13:487-507

29. Wegner JL, Yao MM, Bhullar SK (2009) Dynamic wave soil structure interaction analysis of a two way asymmetric building system DSSIA-3D. J Eng Technol Res 2:26-38

30. Wolf JP (1985) Dynamic soil-structure interaction. Prentice-Hall, Englewood Cliffs

31. Yamashita K, Hamada J, Yamada T (2010) Field Measurements On Piled Rafts with Grid-Form Deep Mixing Walls on Soft Ground, Geaotec Eng-SEAGS 42(2) 